\title{
Detection of interactions via generalized factorial cumulants in systems in and out of equilibrium
}

\author{
Philipp Stegmann, ${ }^{1, *}$ Björn Sothmann, ${ }^{2}$ Alfred Hucht, ${ }^{1}$ and Jürgen König ${ }^{1}$ \\ ${ }^{1}$ Theoretische Physik, Universität Duisburg-Essen and CENIDE, D-47048 Duisburg, Germany \\ ${ }^{2}$ Département de Physique Théorique, Université de Genève, CH-1211 Genève 4, Switzerland
}

(Dated: August 21, 2018)

\begin{abstract}
We introduce time-dependent, generalized factorial cumulants $C_{s}^{m}(t)$ of the full counting statistics of electron transfer as a tool to detect interactions in nanostructures. The violation of the sign criterion $(-1)^{m-1} C_{s}^{m}(t) \geq 0$ for any time $t$, order $m$, and parameter $s$ proves the presence of interactions. For given system parameters, there is a minimal time span $t_{\min }$ and a minimal order $m$ to observe the violation of the sign criterion. We demonstrate that generalized factorial cumulants are more sensitive to interactions than ordinary ones and can detect interactions even in regimes where ordinary factorial cumulants fail. We illustrate our findings with the example of a quantum dot tunnel coupled to electronic reservoirs either in or out of equilibrium.
\end{abstract}

PACS numbers: 73.23.Hk, 02.50.Ey, 72.70.+m, 73.63.Kv

\section{INTRODUCTION}

The stochastic nature of electron transfer in mesoscopic conductors gives rise to both thermal and shot noise. $^{1}$ A deviation of the shot-noise power from the value expected for Poissonian processes of uncorrelated charge transfer indicates correlations. In particular, an enhanced, super-Poissonian shot-noise power is a clear signature of the presence of interactions. ${ }^{2,3}$ Various scenarios for super-Poissonian shot noise of currents sustained by electron tunneling have been studied both theoretically $^{4-10}$ and experimentally. ${ }^{11-17}$

Full information about the counting statistics of charge transfer ${ }^{18,19}$ is contained in the probability distribution $P_{N}(t)$ that $N$ charges have been transferred through the system in time $t$. From its Laplace transform, the moment-generating function $\mathcal{M}(z, t):=\sum_{N} e^{N z} P_{N}(t)$, one can derive moments $M^{m}(t):=\left.\partial_{z}^{m} \mathcal{M}(z, t)\right|_{z=0}$ and cumulants $C^{m}(t):=\left.\partial_{z}^{m} \ln \mathcal{M}(z, t)\right|_{z=0}$ as the $m$ th derivative $(m \geq 1)$ with respect to $z$ taken at $z=0$. The $m$ th moment is the expectation value of the $m$ th power, $M^{m}(t)=\left\langle N^{m}\right\rangle(t)$, with $\langle\cdots\rangle:=\sum_{N} \cdots P_{N}(t)$. For at least two reasons, however, it is advantageous to study cumulants $C^{m}(t)=\left\langle\left\langle N^{m}\right\rangle\right\rangle(t)$ instead of moments. ${ }^{20}$ First, if the charge transport can be separated into statistically independent subprocesses, the cumulants of the total transferred charge are simply given by the sum of the cumulants of all channels. Second, in the longtime limit, all the cumulants $C^{m}(t) \propto t$ grow linearly in $t$ while the moments $M^{m}(t) \propto t^{m}$ grow with different powers. ${ }^{19}$ Higher-order cumulants have been calculated for electron transport through various interacting systems. ${ }^{21-38}$ Experimentally, the cumulants of electron tunneling through quantum dots have been measured up to 20th order by monitoring the charge occupancy of the quantum dot via a capacitively coupled quantum point contact. $^{39-47}$

An enhancement of the shot-noise power due to interactions can be quantified by the Fano factor, i.e., the ratio of the second to the first cumulant. This raises the question whether there is additional information about interactions contained in the higher-order cumulants $m \geq 3$. Higher-order cumulants have been shown ${ }^{43}$ to oscillate, as a universal feature, as a function of any system parameter, measurement time $t$, or order $m$, independent of whether or not interactions are present. As an alternative probe of interactions, factorial cumulants have been suggested instead. ${ }^{48,49}$ The latter are defined as cumulants $\left\langle\left\langle N^{(m)}\right\rangle\right\rangle$ of the factorial power $N^{(m)}:=N(N-1) \cdots(N-m+1)$. For noninteracting systems, factorial cumulants do not change sign as a function of any system parameter or time $t$ and display only trivial sign changes $(-1)^{m-1}$ as a function of $m$. Any deviation from this behavior proves the presence of interactions. Interactions are, however, only a necessary but not a sufficient criterion to obtain nontrivial sign changes of factorial cumulants. In fact, the results of a recent measurement of hole transfer through an interacting quantum $\operatorname{dot}^{47}$ could be explained within an effectively noninteracting model.

\section{GENERALIZED FACTORIAL CUMULANTS}

In this paper, we present a more sensitive and versatile indicator of interactions based on generalized factorial cumulants. To this end, we define the generalized factorial-moment generating function

$$
\mathcal{M}_{s}(z, t):=\frac{\sum_{N=0}^{\infty}(z+s)^{N} P_{N}(t)}{\sum_{N=0}^{\infty} s^{N} P_{N}(t)},
$$

where $N$ is the number of transferred charges, counting the charges that, say, leave the central part of the system (e.g., quantum dot) into some leads while not counting those entering; therefore, by definition, $N \geq 0$. From the generating function, we obtain gen- 
eralized factorial moments $M_{s}^{m}(t):=\left.\partial_{z}^{m} \mathcal{M}_{s}(z, t)\right|_{z=0}$ and cumulants $C_{s}^{m}(t):=\left.\partial_{z}^{m} \ln \mathcal{M}_{s}(z, t)\right|_{z=0}$. Defining an $s$-dependent expectation value $\langle\cdots\rangle_{s}(t):=$ $\sum_{N} \cdots s^{N} P_{N}(t) / \sum_{N} s^{N} P_{N}(t)$, one can show that $M_{s}^{m}(t)=\left\langle s^{-m} N^{(m)}\right\rangle_{s}(t)$ and $C_{s}^{m}(t)=\left\langle\left\langle s^{-m} N^{(m)}\right\rangle\right\rangle_{s}(t)$, where $\left\langle\left\langle N^{m}\right\rangle\right\rangle_{s}$ is related to $\left\langle N^{m}\right\rangle_{s}$ via the same recursive relation that relates $\left\langle\left\langle N^{m}\right\rangle\right\rangle$ to $\left\langle N^{m}\right\rangle .^{20,50}$ The factorial cumulants are recovered by setting $s=1$, i.e., $C_{1}^{m}(t)=\left\langle\left\langle N^{(m)}\right\rangle\right\rangle(t)$.

The generalized factorial cumulants with real $s$ can be expressed in terms of the zeros $z_{j}(t)$ of $\mathcal{M}_{s}(z, t)$ with degeneracies $\alpha_{j}$ via the formula ${ }^{51}$

$$
C_{s}^{m}(t)=(-1)^{m-1}(m-1) ! \sum_{j} \frac{\alpha_{j} \cos \left[m \arg \left(-z_{j}\right)\right]}{\left|z_{j}\right|^{m}} .
$$

The zeros are either real or appear in complex conjugated pairs. By varying $s$, their positions are simply shifted in the complex plane, i.e., $z_{j}(s)+s$ is independent of $s$.

For a noninteracting fermionic system, the generating function has the form $\mathcal{M}_{s}(z, t)=\left(\prod_{j}\left[1-p_{j}+p_{j}(z+\right.\right.$ $s)]) / \sum_{N} s^{N} P_{N}(t)$, where $p_{j}$ with $0 \leq p_{j} \leq 1$ is the probability for a single-particle transfer to occur. ${ }^{52,53}$ Hence, all zeros $z_{j}=1-1 / p_{j}-s$ lie on the real axis with $-z_{j} \geq s$ and positive $\alpha_{j}$. If $s \geq 0$, then $\arg \left(-z_{j}\right)=0$, which fixes the sign of all generalized factorial cumulants,

$$
(-1)^{m-1} C_{s}^{m}(t) \geq 0 .
$$

If $s<0$, this criterion still holds for all even orders $m$. Any violation of this behavior is a clear indication of the presence of interactions. Equation (3), which holds for any noninteracting fermionic system constitutes the main result of our paper. For $s=1$ and $m=2$, it reduces to the well-known criterion that a super-Poissonian Fano factor, $C^{2}(t) / C^{1}(t)>1$, indicates interactions.

What are the conditions on $t, m$, and $s$ to observe a violation of Eq. (3) and thus prove the presence of interactions for a given system? First, at very short times $t$, all charge-transfer processes become independent from each other. Thus, all zeros of $\mathcal{M}_{s}(z, t)$ are on the real axis and Eq. (3) holds. As a consequence, $t$ must be larger than a minimal time span $t_{\text {min }}$. Second, higherorder generalized factorial cumulants are favorable since in Eq. (2) deviations of $\arg \left(-z_{j}\right)$ from 0 are amplified by the multiplication with $m$ such that $\cos \left[m \arg \left(-z_{j}\right)\right]$ can become negative. Third, with decreasing $s$, the zeros $z_{j}(s)$ are shifted towards the positive real direction in the complex plane. Again, deviations of $\arg \left(-z_{j}\right)$ from 0 become larger. Moreover, Eq. (2) is dominated by the zeros with the largest $\left|z_{j}(s)\right|^{-m}$. Thus, by varying $s$, different zeros can be brought into focus.

\section{EXAMPLES}

In the following, we illustrate our findings on a singlelevel quantum dot tunnel coupled to electronic reservoirs either in or out of equilibrium (cf. Fig. 1). A large Coulomb interaction ensures that double occupation of the dot is forbidden. For weak tunneling (sequential tunneling), electron transfer through the dot is described by the master equation [see Fig. 1 (c)]

$$
\begin{aligned}
& \dot{P}_{N}^{0}(t)=-\left(\gamma_{\uparrow 0}+\gamma_{\downarrow 0}\right) P_{N}^{0}(t)+\gamma_{0 \uparrow} P_{N-1}^{\uparrow}(t)+\gamma_{0 \downarrow} P_{N-1}^{\downarrow}(t), \\
& \dot{P}_{N}^{\uparrow}(t)=\gamma_{\uparrow 0} P_{N}^{0}(t)-\gamma_{0 \uparrow} P_{N}^{\uparrow}(t), \\
& \dot{P}_{N}^{\downarrow}(t)=\gamma_{\downarrow 0} P_{N}^{0}(t)-\gamma_{0 \downarrow} P_{N}^{\downarrow}(t),
\end{aligned}
$$

for the probability $P_{N}^{\chi}(t)$ that $N$ electrons have left the quantum dot in time $t$ and the dot is in a state $\chi=0, \uparrow, \downarrow$ (electrons entering the dot are not counted, i.e., $N$ is not the net charge transfer between the dot and some lead). The transition rates $\gamma_{\chi \chi^{\prime}}$ from state $\chi^{\prime}$ to $\chi$ are given by Fermi's golden rule. Making use of the $z$-transform $P_{z}^{\chi}(t):=\sum_{N} z^{N} P_{N}^{\chi}(t)$, we obtain

$$
\dot{\mathbf{P}}_{z}(t)=\mathbf{W}_{z} \mathbf{P}_{z}(t),
$$

with the vector $\mathbf{P}_{z}=\left(P_{z}^{0}, P_{z}^{\uparrow}, P_{z}^{\downarrow}\right)^{T}$ and the matrix

$$
\mathbf{W}_{z}=\left(\begin{array}{ccc}
-\gamma_{\uparrow 0}-\gamma_{\downarrow 0} & z \gamma_{0 \uparrow} & z \gamma_{0 \downarrow} \\
\gamma_{\uparrow 0} & -\gamma_{0 \uparrow} & 0 \\
\gamma_{\downarrow 0} & 0 & -\gamma_{0 \downarrow}
\end{array}\right)
$$

The solution of Eq. (5) is $\mathbf{P}_{z}(t)=\exp \left(\mathbf{W}_{z} t\right) \mathbf{P}(0)$, where $\mathbf{P}(0)$ is the initial probability distribution. Assuming that electron counting starts when the system has reached its steady state, $\mathbf{P}(0)$ is given by the stationary probability distribution, determined by $\mathbf{W}_{1} \mathbf{P}(0)=$ 0 and $\mathbf{e}^{T} \cdot \mathbf{P}(0)=1$, with $\mathbf{e}^{T}=(1,1,1)$. The moment-generating function $\mathcal{M}_{s}(z, t)=P_{z+s}(t) / P_{s}(t)$, with $P_{z}(t)=\mathbf{e}^{T} \cdot \mathbf{P}_{z}(t)$, can be expressed in terms of the three eigenvalues $\lambda_{j, z}(j=1,2,3)$ of the matrix $\mathbf{W}_{z}=\sum_{j} \lambda_{j, z} \mathbf{r}_{j, z} \otimes \mathbf{l}_{j, z}^{T}$ by making use of the decomposition into left and right eigenvectors $\mathbf{l}_{j, z}$ and $\mathbf{r}_{j, z}$ with

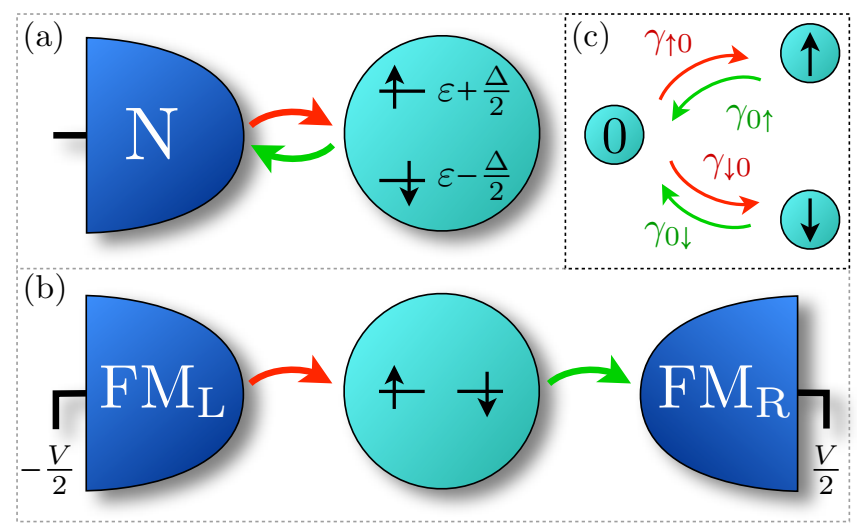

FIG. 1. (Color online) (a) Equilibrium scenario: A singlelevel quantum dot subject to a Zeeman field is tunnel coupled to one normal lead. (b) Nonequilibrium scenario: A single-level quantum dot is tunnel coupled to two ferromagnetic leads with finite bias voltage. (c) Sketch of the states and transition rates. 

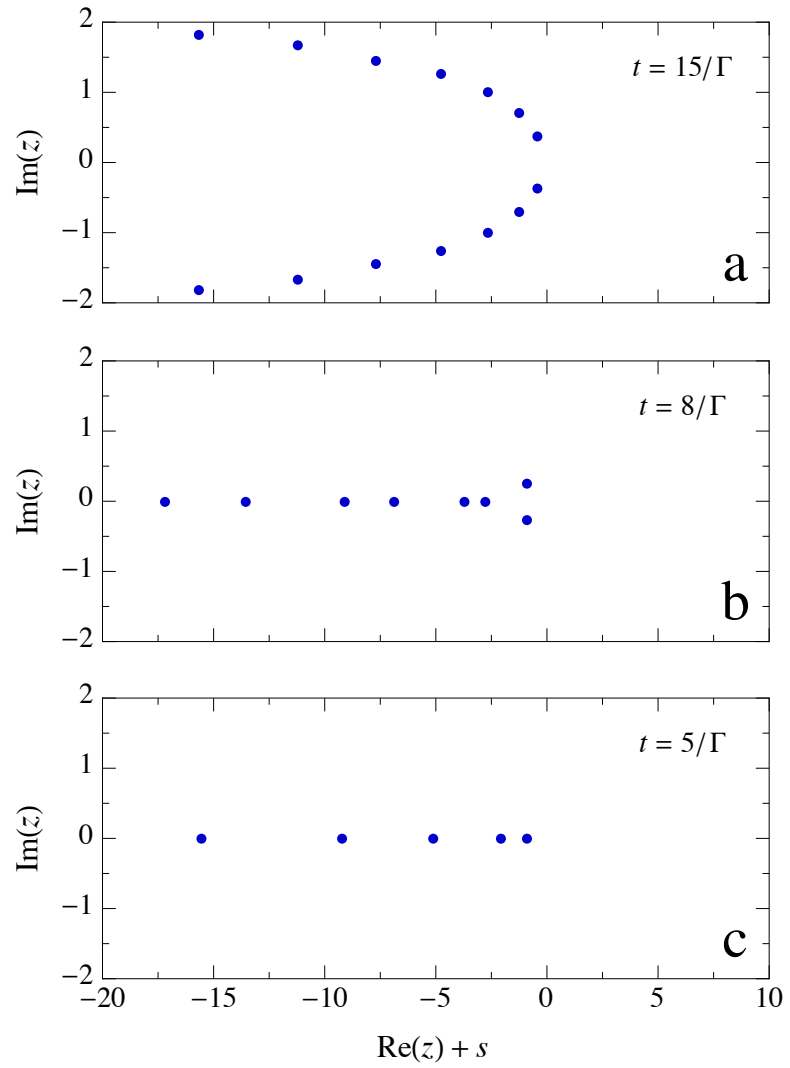

FIG. 2. (Color online) Zeros $z_{j}$ for the equilibrium scenario with $\Delta=k_{\mathrm{B}} T / 2, \varepsilon=-\Delta$, and times $t=5 / \Gamma, 8 / \Gamma, 15 / \Gamma$. For times $t \leq t_{\min } \approx 6.83 / \Gamma$, the zeros remain on the real axis. For $t>t_{\min }$, zeros leave the real axis and interactions can be detected by generalized factorial cumulants.

normalization $\mathbf{l}_{j, z}^{T} \cdot \mathbf{r}_{j^{\prime}, z}=\delta_{j j^{\prime}}$. We find

$$
\mathcal{M}_{s}(z, t)=\sum_{j=1}^{3} c_{j, z+s} \exp \left(\lambda_{j, z+s} t\right) / P_{s}(t),
$$

where we defined the amplitudes $c_{j, z}:=$ $\left(\mathbf{e}^{T} \cdot \mathbf{r}_{j, z}\right)\left(\mathbf{l}_{j, z} \cdot \mathbf{P}(0)\right)$. The generalized factorial cumulants are then evaluated by performing derivatives of $\ln \mathcal{M}_{s}(z, t)$ with respect to $z$ at $z=0$.

\section{A. Equilibrium scenario}

As a first example, we consider an equilibrium scenario with a quantum dot coupled to one normal lead [see Fig. 1 (a)]. Spin degeneracy of the dot level is lifted by a Zeeman field, $\varepsilon_{\sigma}=\varepsilon \pm \Delta / 2$, where the quantum-dot level $\varepsilon$ measured relative to the Fermi energy of the lead may be tuned by a gate voltage. The positive (negative) sign corresponds to $\sigma=\uparrow(\downarrow)$. Fermi's golden rule yields $\gamma_{\sigma 0}=\Gamma f\left(\varepsilon_{\sigma}\right)$ and $\gamma_{0 \sigma}=\Gamma\left[1-f\left(\varepsilon_{\sigma}\right)\right]$ with the Fermi function $f_{\sigma}=\left[1+\exp \left(\varepsilon_{\sigma} / k_{\mathrm{B}} T\right)\right]^{-1}$ and tunnel-coupling strength $\Gamma$. Weak tunneling corresponds to $\hbar \Gamma \ll k_{\mathrm{B}} T$.
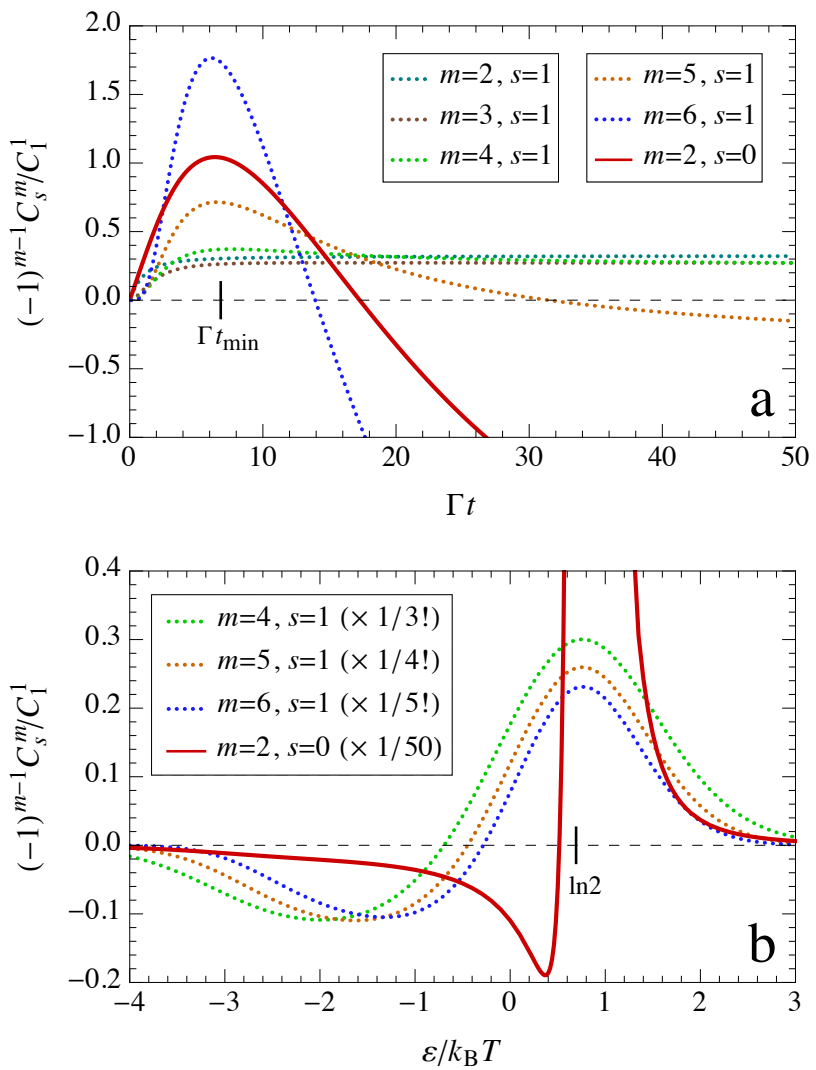

FIG. 3. (Color online) (Generalized) factorial cumulant $C_{s}^{m}$ normalized by $C_{1}^{1}=C^{1}=\langle N\rangle>0$ for the equilibrium scenario as a function of (a) time $t$ and (b) dot-level energy $\varepsilon$. The parameters are $\Delta=k_{\mathrm{B}} T / 2$ and (a) $\varepsilon=-\Delta$ or (b) $t=100 / \Gamma$. Negative values of $(-1)^{m-1} C_{s}^{m}(t)$ indicate the presence of interactions. Interactions can be detected (a) for times larger than $t_{\min } \approx 6.83 / \Gamma$ and $(\mathrm{b})$ for level positions $\varepsilon \lesssim k_{\mathrm{B}} T \ln 2$.

For a vanishing Zeeman field, $\Delta=0$, the model can be mapped onto a noninteracting one in which a single, spinless quantum-dot level $(\chi=0,1)$ is filled with rate $\gamma_{10}:=\gamma_{\uparrow 0}+\gamma_{\downarrow 0}$ and emptied with rate $\gamma_{01}:=\gamma_{0 \uparrow}=\gamma_{0 \downarrow}$. Only two $c_{j, z}$ are nonvanishing. As a consequence, all zeros of $\mathcal{M}_{s}(z, t)$ lie on the real axis and Eq. (3) holds. For a finite Zeeman field, $\Delta \neq 0$, the zeros of $\mathcal{M}_{s}(z, t)$ remain on the real axis for short times. ${ }^{54}$ However, after a minimal time span $t_{\text {min }}$, which depends on the system parameters and can be larger or smaller than $1 / \Gamma$, the first pair of zeros moves from the real axis into the complex plane (cf. Fig. 2). Beyond this time, the presence of interactions can be detected from the full counting statistics, as we will detail in the following.

In Fig. 3 (a), we show the factorial cumulants $(s=1)$ as a function of time for fixed values of $\varepsilon / k_{\mathrm{B}} T$ and $\Delta / k_{\mathrm{B}} T$. While for the first four generalized factorial cumulants Eq. (3) holds, there is a sign change for higher orders $m$, which indicates the presence of interactions. With increasing $m$, the time at which the sign change 

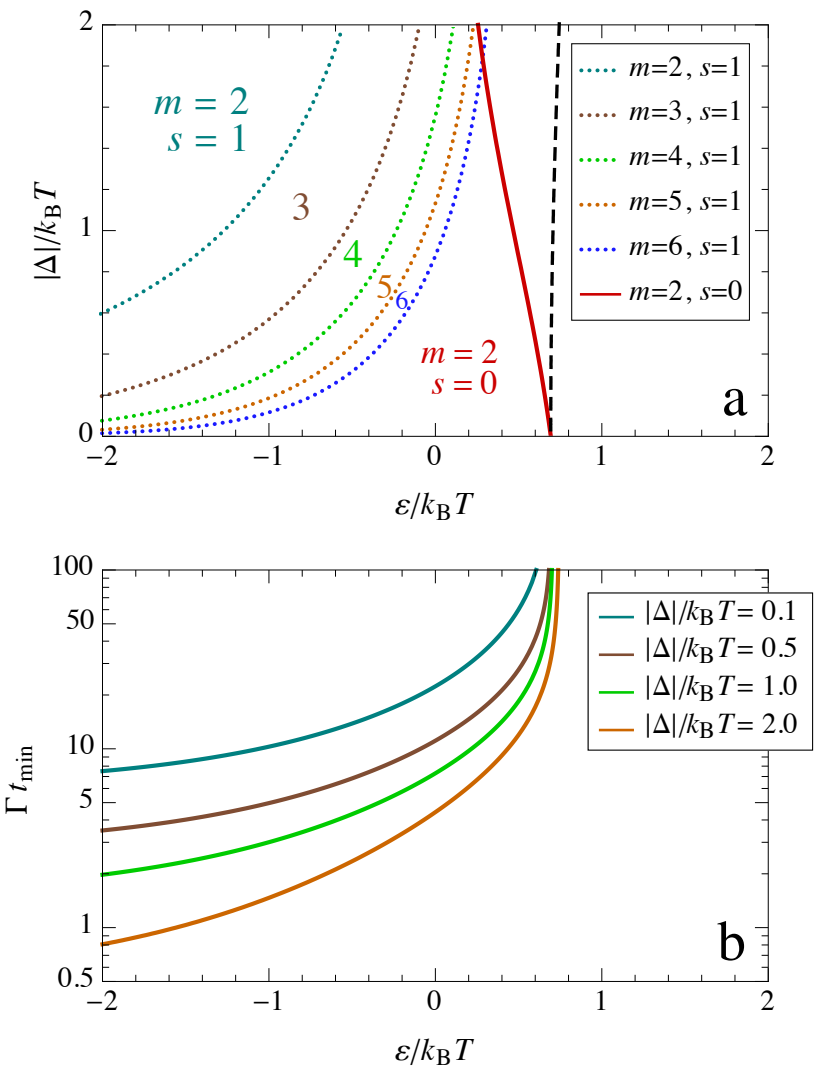

FIG. 4. (Color online) (a) Parameter space and (b) minimal time span $t_{\min }$ of the system in equilibrium. (a) To the left of the colored lines, the sign criterion for the respective $C_{s}^{m}(t)$ is violated at some time $t$. To the right of the dashed line, $\mathcal{M}_{s}(z, t)$ has only real zeros such that the sign criterion cannot be violated. (b) Minimal time span $t_{\min }$ increases with increasing $\varepsilon$ and decreasing $|\Delta|$ and diverges at the dashed line in (a) and for $\Delta=0$.

occurs decreases and approaches $t_{\min }$. However, when considering generalized factorial cumulants, we observe that already $C_{0}^{2}(t)$ violates the sign criterion Eq. (3). In Fig. 3 (b), the gate-voltage dependence of the factorial cumulants is depicted. We find that the criterion Eq. (3) is violated for low-lying level energies $\varepsilon$, indicating that interactions are more important in the regime when both spin states in the quantum dot have a finite occupation probability. Again, for generalized factorial cumulants with $s=0$, the possibility to detect the presence of interactions is dramatically enhanced.

Figure 4 (a) illustrates the possibility to detect interactions via different generalized factorial cumulants. To the right of the dashed line, given by $\frac{\varepsilon}{k_{\mathrm{B}} T}=\ln 2+$ $\left(\frac{\Delta}{k_{\mathrm{B}} T}\right)^{2} / 72+\mathcal{O}\left(\frac{\Delta}{k_{\mathrm{B}} T}\right)^{4}$, all zeros of $\mathcal{M}_{s}(z, t)$ remain on the real axis [see Fig. 5 (a)] and $t_{\text {min }}$ is infinite [see Fig. 4 (b)]. Therefore, a violation of the sign criterion Eq. (3) and thus a detection of interactions is only possible for $\varepsilon \lesssim k_{\mathrm{B}} T \ln 2$. The second-order factorial cumulant, $s=1$ and $m=2$, violates Eq. (3) only for
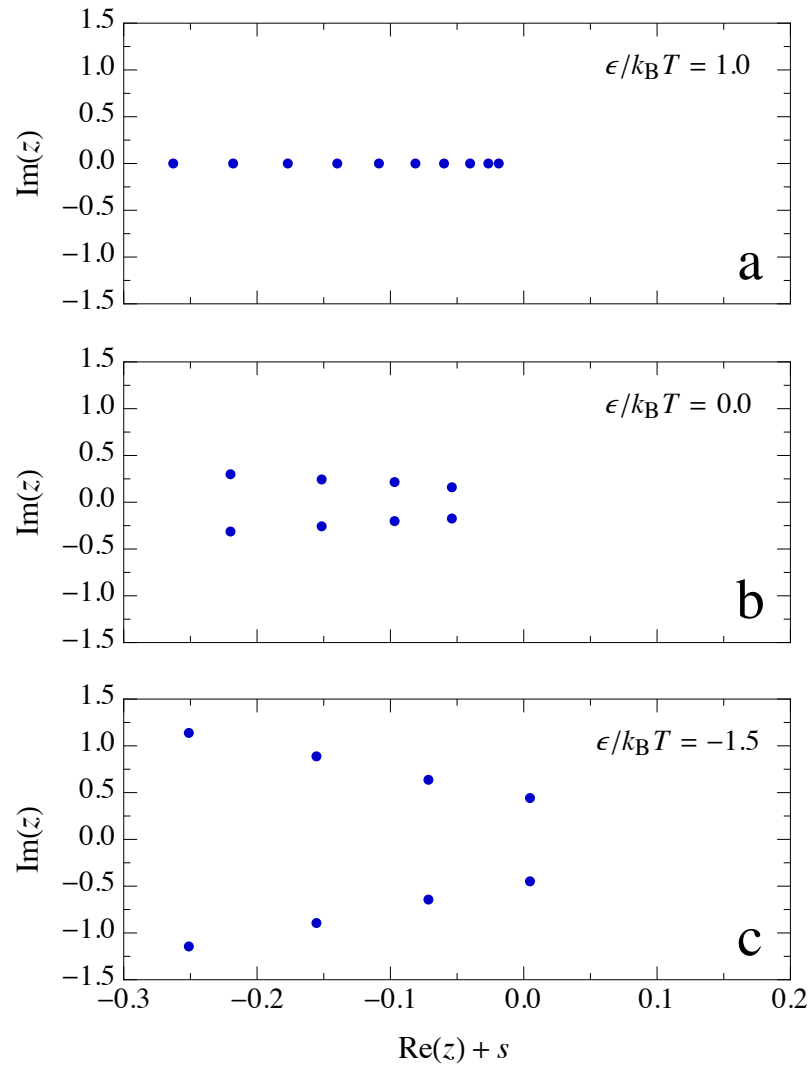

FIG. 5. (Color online) Zeros $z_{j}$ for the equilibrium scenario with $\Delta=k_{\mathrm{B}} T / 2, t=100 / \Gamma$, and $\varepsilon / k_{\mathrm{B}} T=-1.5,0.0,1.0$. For $\varepsilon / k_{\mathrm{B}} T \lesssim \ln 2$, zeros leave the real axis after some time and interactions can be detected by generalized factorial cumulants.

rather large values of the Zeeman energy and a low-lying quantum-dot level. With increasing $m$, the region in which interactions can be detected is gradually increased to lower Zeeman splitting $|\Delta|$ and larger level positions $\varepsilon$. For generalized factorial cumulants with $s=0$, already the second order, $m=2$, covers a much larger region of violation of Eq. (3). This clearly demonstrates the enhanced sensitivity of generalized factorial cumulants to the presence of interactions.

\section{B. Nonequilibrium scenario}

As a second example [see Fig. 1 (b)], we consider a spin-degenerate quantum dot, $\Delta=0$, coupled to two ferromagnetic leads with parallel magnetizations and tunnel couplings $\Gamma_{r}, r=\mathrm{L}, \mathrm{R}$. A finite bias voltage $V$ applied symmetrically between the two ferromagnets gives rise to a nonequilibrium scenario. Each ferromagnet is characterized by its spin polarization $p_{r}$ ranging from $p_{r}=0$ for a normal metal to $p_{r}=1$ for a halfmetallic ferromagnet with majority spins only. In the limit $|\varepsilon \pm e V / 2| \gg k_{\mathrm{B}} T, \Gamma$, transport through the quantum dot is supported by unidirectional sequential tun- 

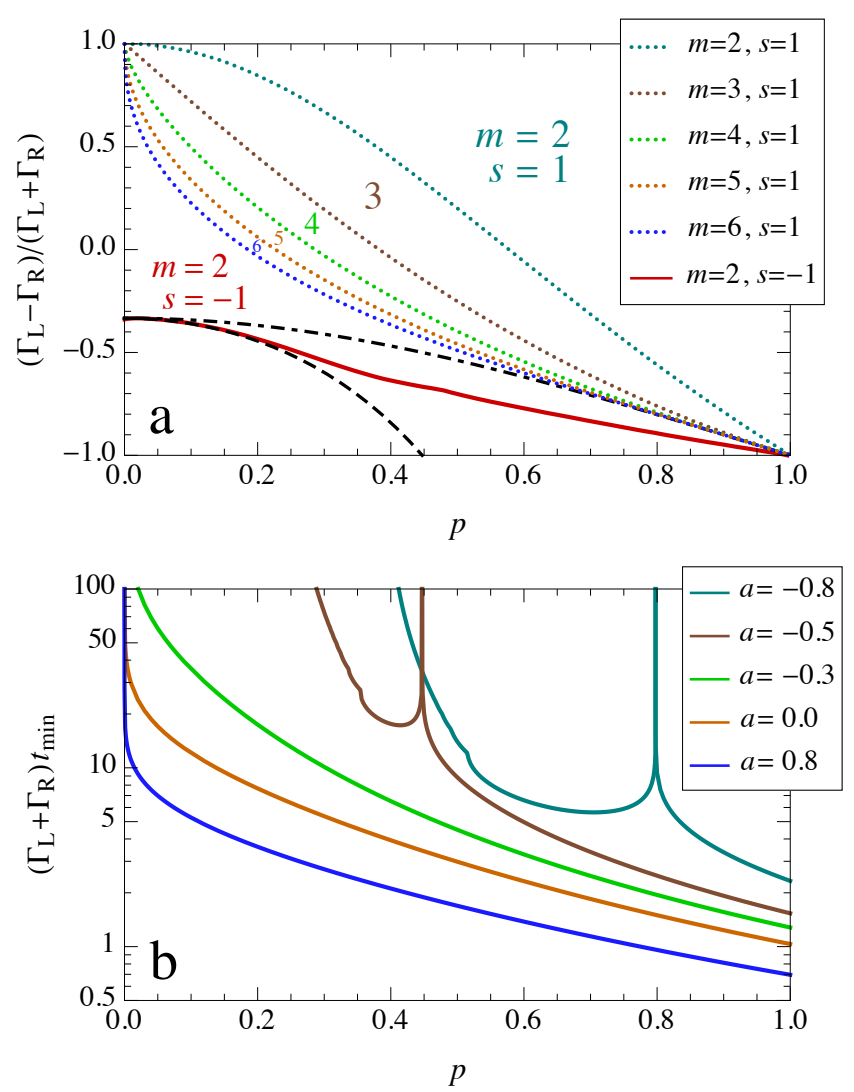

FIG. 6. (Color online) (a) Parameter space and (b) minimal time span $t_{\min }$ of the system out of equilibrium. The polarizations are $p_{\mathrm{L}}=p_{\mathrm{R}}=p$, however, a different polarization $p_{\mathrm{L}}$ (even $p_{\mathrm{L}}=0$ ) does not change the results qualitatively. (a) Above the colored lines, the sign criterion for the respective $C_{s}^{m}(t)$ is violated at some time $t$. Below the dashed line, $\mathcal{M}_{s}(z, t)$ has only real zeros such that the sign criterion cannot be violated. (b) For $a \geq-1 / 3$, the minimal time $t_{\text {min }}$ increases with decreasing $p$ and decreasing $a$ and diverges at $p=0$; for $a<-1 / 3$, the minimal time $t_{\text {min }}$ diverges both at the dashed and the dotted-dashed line in (a).

neling with rates $\gamma_{\uparrow 0}=\left(1+p_{\mathrm{L}}\right) \Gamma_{\mathrm{L}}, \gamma_{\downarrow 0}=\left(1-p_{\mathrm{L}}\right) \Gamma_{\mathrm{L}}$, $\gamma_{0 \uparrow}=\left(1+p_{\mathrm{R}}\right) \Gamma_{\mathrm{R}}$, and $\gamma_{0 \downarrow}=\left(1-p_{\mathrm{R}}\right) \Gamma_{\mathrm{R}}$. We denote the asymmetry of tunnel couplings to source and drain by $a=\left(\Gamma_{\mathrm{L}}-\Gamma_{\mathrm{R}}\right) /\left(\Gamma_{\mathrm{L}}+\Gamma_{\mathrm{R}}\right)$.

In Fig. 6 (a), we demonstrate the possibility to detect the presence of interactions in this nonequilibrium scenario. Below the dashed line, given by $a=$ $\left(3 p_{\mathrm{R}}^{2}+4 p_{\mathrm{L}} p_{\mathrm{R}}+1\right) /\left(3 p_{\mathrm{R}}^{2}-3\right)$, all zeros of $\mathcal{M}_{s}(z, t)$ remain on the real axis [see Fig. 7 (c)], i.e., neither factorial nor generalized factorial cumulants indicate the presence of interactions. The same is true for the trivial case $p_{\mathrm{R}}=0$ and the dotted-dashed line, given by $a=\left(p_{\mathrm{R}}^{2}+2 p_{\mathrm{L}} p_{\mathrm{R}}+1\right) /\left(p_{\mathrm{R}}^{2}-2 p_{\mathrm{L}} p_{\mathrm{R}}-3\right)$, where the system can be mapped onto a noninteracting Hamiltonian, described by a two-state model. Of course, $t_{\text {min }}$ diverges at these system parameters [see Fig. 6 (b)]. Above the dotted-dashed line, all the zeros move into the complex plane [see Fig. 7 (a)], including the rightmost zeros which
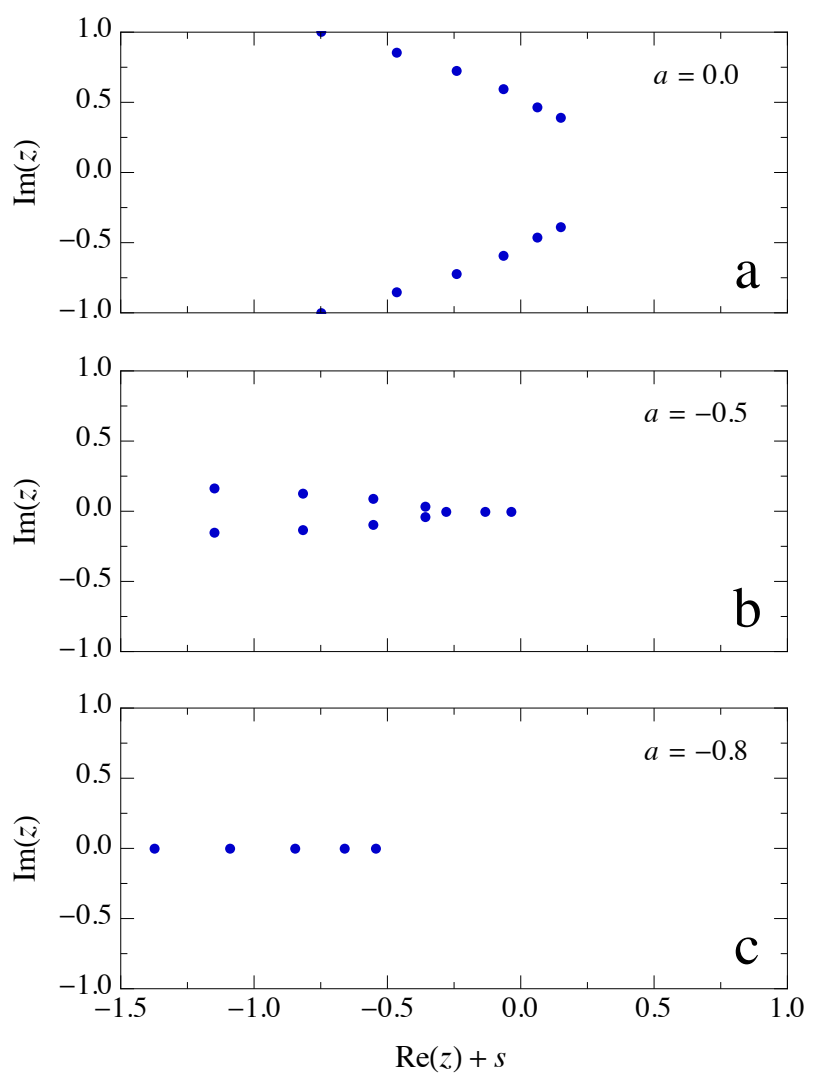

FIG. 7. (Color online) Zeros $z_{j}$ for the nonequilibrium scenario discussed in Fig. 6 with $p_{\mathrm{L}}=p_{\mathrm{R}}=0.35, t=$ $50 /\left(\Gamma_{\mathrm{L}}+\Gamma_{\mathrm{R}}\right)$, and $a=\left(\Gamma_{\mathrm{L}}-\Gamma_{\mathrm{R}}\right) /\left(\Gamma_{\mathrm{L}}+\Gamma_{\mathrm{R}}\right)=-0.8,-0.5,0.0$. For $a=-0.8$, all zeros remain on and for $a=0.0$ all zeros leave the real axis. For $a=-0.5$, the rightmost zeros remain on the real axis, but zeros further to the left move into the complex plane. While factorial cumulants are insensitive to interactions in this case, generalized factorial cumulants can detect their presence.

dominate the behavior of the factorial cumulants, $s=1$. For large values of the spin polarization $p=p_{\mathrm{L}}=p_{\mathrm{R}}$ or the asymmetry $a$ of the tunnel couplings, Eq. (3) is already violated for the second-order factorial cumulant $(m=2)$. With decreasing $p$ or $a$, higher orders $m$ of the factorial cumulants are needed to detect interaction.

An interesting regime resides between the dashed and dotted-dashed line. Here, the rightmost zeros remain on the real axis, but zeros further to the left move into the complex plane [see Fig. 7 (b)]. As a consequence, a violation of Eq. (3) cannot be found for any ordinary $(s=1)$ factorial cumulant. This limitation is overcome by generalized factorial cumulants. By an appropriate choice of $s$, they can probe the position of any zero of $\mathcal{M}_{s}(z, t)$ in the complex plane and thus detect interactions where ordinary factorial cumulants fail. Furthermore, as in the equilibrium case, for a given order $m$, generalized factorial cumulants can detect interactions in a larger area of parameter space. 


\section{CONCLUSIONS}

In summary, we proposed generalized factorial cumulants $C_{s}^{m}(t)$ of the full counting statistics of electron transport through nanostructures as a sensitive and versatile tool to detect the presence of interactions via the violation of Eq. (3). We found that generalized factorial cumulants are superior to ordinary ones: With decreasing $s$, interaction effects already show up in lower order $m$ and at earlier times. This may be crucial for overcoming experimental limitations. Furthermore, there are regimes in which general factorial cumulants can detect interactions while ordinary ones completely fail. We illustrated our theoretical findings with two examples of a quantum dot tunnel coupled to electronic reservoirs. Importantly, we demonstrated that, already in a simple equilibrium case of experimental relevance, interactions can be detected via generalized factorial cumulants. Finally, we emphasize the general validity of the criterion Eq. (3). It is not restricted to any specific type of interaction or transport regime and also covers multilevel and multichannel setups. It also applies to systems where coherences described by off-diagonal elements of the reduced density matrix have to be taken into account.

\section{ACKNOWLEDGMENTS}

We acknowledge financial support from the Swiss National Science Foundation and the DFG under project KO $1987 / 5$.
* philipp.stegmann@uni-due.de

1 Y. M. Blanter and M. Büttiker, Shot noise in mesoscopic conductors, Phys. Rep. 336, 1 (2000).

${ }^{2}$ G. B. Lesovik, Excess quantum noise in $2 \mathrm{D}$ ballistic point contacts, JETP Lett. 49, 592 (1989).

3 M. Büttiker, Scattering theory of thermal and excess noise in open conductors, Phys. Rev. Lett. 65, 2901 (1990).

4 A. Cottet, W. Belzig, and C. Bruder, Positive Cross Correlations in a Three-Terminal Quantum Dot with Ferromagnetic Contacts, Phys. Rev. Lett. 92, 206801 (2004).

5 H.-A. Engel and D. Loss, Asymmetric Quantum Shot Noise in Quantum Dots, Phys. Rev. Lett. 93, 136602 (2004).

6 T. Novotný, A. Donarini, C. Flindt, and A.-P. Jauho, Shot Noise of a Quantum Shuttle, Phys. Rev. Lett. 92, 248302 (2004).

7 J. Koch and F. von Oppen, Franck-Condon Blockade and Giant Fano Factors in Transport through Single Molecules, Phys. Rev. Lett. 94, 206804 (2005).

8 A. Thielmann, M. H. Hettler, J. König, and G. Schön, Cotunneling Current and Shot Noise in Quantum Dots, Phys. Rev. Lett. 95, 146806 (2005).

9 R. Sánchez, G. Platero, and T. Brandes, Resonance Fluorescence in Transport through Quantum Dots: Noise Properties, Phys. Rev. Lett. 98, 146805 (2007).

10 B. Sothmann, J. König, and Y. Gefen, Mesoscopic Stoner Instability in Metallic Nanoparticles Revealed by Shot Noise, Phys. Rev. Lett. 108, 166603 (2012).

11 S. S. Safonov, A. K. Savchenko, D. A. Bagrets, O. N. Jouravlev, Y. V. Nazarov, E. H. Linfield, and D. A. Ritchie, Enhanced Shot Noise in Resonant Tunneling via Interacting Localized States, Phys. Rev. Lett. 91, 136801 (2003).

12 E. Onac, F. Balestro, B. Trauzettel, C. F. J. Lodewijk, and L. P. Kouwenhoven, Shot-Noise Detection in a Carbon Nanotube Quantum Dot, Phys. Rev. Lett. 96, 026803 (2006).

13 P. Barthold, F. Hohls, N. Maire, K. Pierz, and R. J. Haug, Enhanced Shot Noise in Tunneling through a Stack of Coupled Quantum Dots, Phys. Rev. Lett. 96, 246804 (2006).

14 Y. Chen and R. A. Webb, Positive Current Correlations Associated with Super-Poissonian Shot Noise, Phys. Rev. Lett. 97, 066604 (2006).
15 O. Zarchin, Y. C. Chung, M. Heiblum, D. Rohrlich, and V. Umansky, Electron Bunching in Transport through Quantum Dots in a High Magnetic Field, Phys. Rev. Lett. 98, 066801 (2007).

16 Y. Zhang, L. DiCarlo, D. T. McClure, M. Yamamoto, S. Tarucha, C. M. Marcus, M. P. Hanson, and A. C. Gossard, Noise Correlations in a Coulomb-Blockaded Quantum Dot, Phys. Rev. Lett. 99, 036603 (2007).

17 G. Kießlich, E. Schöll, T. Brandes, F. Hohls, and R. J. Haug, Noise Enhancement due to Quantum Coherence in Coupled Quantum Dots, Phys. Rev. Lett. 99, 206602 (2007).

18 L. S. Levitov, H. Lee, and G. B. Lesovik, Electron counting statistics and coherent states of electric current, J. Math. Phys. 37, 4845 (1996).

19 D. A. Bagrets and Yu. V. Nazarov, Full counting statistics of charge transfer in Coulomb blockade systems, Phys. Rev. B 67, 085316 (2003).

20 As usual, $\langle\langle\cdots\rangle\rangle$ is defined by the linearity property $\left\langle\left\langle\alpha N^{m}+\beta N^{n}\right\rangle\right\rangle=\alpha\left\langle\left\langle N^{m}\right\rangle\right\rangle+\beta\left\langle\left\langle N^{n}\right\rangle\right\rangle$ and its relation to $\langle\cdots\rangle$ via the recursive relation $\left\langle\left\langle N^{m}\right\rangle\right\rangle=\left\langle N^{m}\right\rangle-$ $\sum_{j=1}^{m-1}\left(\begin{array}{c}m-1 \\ j-1\end{array}\right)\left\langle\left\langle N^{j}\right\rangle\right\rangle\left\langle N^{m-j}\right\rangle$ for $m \geq 1$.

21 J. Börlin, W. Belzig, and C. Bruder, Full Counting Statistics of a Superconducting Beam Splitter, Phys. Rev. Lett. 88, 197001 (2002).

22 S. Pilgram and M. Büttiker, Statistics of charge fluctuations in chaotic cavities, Phys. Rev. B 67, 235308 (2003).

23 J. C. Cuevas and W. Belzig, Full Counting Statistics of Multiple Andreev Reflections, Phys. Rev. Lett. 91, 187001 (2003).

24 G. Johansson, P. Samuelsson, and A. Ingerman, Full Counting Statistics of Multiple Andreev Reflection, Phys. Rev. Lett. 91, 187002 (2003).

25 L. S. Levitov and M. Reznikov, Counting statistics of tunneling current, Phys. Rev. B 70, 115305 (2004).

26 W. Belzig, Full counting statistics of super-Poissonian shot noise in multilevel quantum dots, Phys. Rev. B 71, 161301(R) (2005).

27 A. Braggio, J. König, and R. Fazio, Full Counting Statistics in Strongly Interacting Systems: Non-Markovian Effects, Phys. Rev. Lett. 96, 026805 (2006). 
28 A. O. Gogolin and A. Komnik, Towards full counting statistics for the Anderson impurity model, Phys. Rev. B 73, 195301 (2006).

29 C. Emary, D. Marcos, R. Aguado, and T. Brandes, Frequency-dependent counting statistics in interacting nanoscale conductors, Phys. Rev. B 76, 161404(R) (2007).

30 C. Flindt, T. Novotný, A. Braggio, M. Sassetti, and A.P. Jauho, Counting Statistics of Non-Markovian Quantum Stochastic Processes, Phys. Rev. Lett. 100, 150601 (2008).

31 D. Urban, J. König, and R. Fazio, Coulomb-interaction effects in full counting statistics of a quantum-dot AharonovBohm interferometer, Phys. Rev. B 78, 075318 (2008).

32 S. Lindebaum, D. Urban, and J. König, Spin-induced charge correlations in transport through interacting quantum dots with ferromagnetic leads, Phys. Rev. B 79, 245303 (2009).

33 T. L. Schmidt and A. Komnik, Charge transfer statistics of a molecular quantum dot with a vibrational degree of freedom, Phys. Rev. B 80, 041307(R) (2009).

34 C. Flindt, T. Novotný, A. Braggio, and A.-P. Jauho, Counting statistics of transport through Coulomb blockade nanostructures: High-order cumulants and non-Markovian effects, Phys. Rev. B 82, 155407 (2010).

35 G. Schaller, G. Kießlich, and T. Brandes, Counting statistics in multistable systems, Phys. Rev. B 81, 205305 (2010).

36 F. Domínguez, G. Platero, and S. Kohler, Electron bunching in triple quantum dot interferometers, Chem. Phys. 375, 284 (2010).

37 D. F. Urban, R. Avriller, and A. Levy Yeyati, Nonlinear effects of phonon fluctuations on transport through nanoscale junctions, Phys. Rev. B 82, 121414(R) (2010).

38 A. Braggio, M. Governale, M. G. Pala, and J. König, Superconducting proximity effect in interacting quantum dots revealed by shot noise, Solid State Commun. 151, 155 (2011).

39 S. Gustavsson, R. Leturcq, B. Simovič, R. Schleser, T. Ihn, P. Studerus, K. Ensslin, D. C. Driscoll, and A. C. Gossard, Counting Statistics of Single Electron Transport in a Quantum Dot, Phys. Rev. Lett. 96, 076605 (2006).

40 T. Fujisawa, T. Hayashi, R. Tomita, and Y. Hirayama, Bidirectional Counting of Single Electrons, Science 312, 1634 (2006).

41 S. Gustavsson, R. Leturcq, T. Ihn, K. Ensslin, M. Reinwald, and W. Wegscheider, Measurements of higher-order noise correlations in a quantum dot with a finite bandwidth detector, Phys. Rev. B 75, 075314 (2007).

42 C. Fricke, F. Hohls, W. Wegscheider, and R. J. Haug, Bimodal counting statistics in single-electron tunneling through a quantum dot, Phys. Rev. B 76, 155307 (2007).

43 C. Flindt, C. Fricke, F. Hohls, T. Novotný, K. Netočný, T. Brandes, and R. J. Haug, Universal oscillations in counting statistics, PNAS 106, 10116 (2009).

44 S. Gustavsson, M. Leturcq, R. Studer, I. Shorubalko, T. Ihn, K. Ensslin, D. C. Driscoll, and A. C. Gossard, Electron counting in quantum dots, Surf. Sci. Rep. 64, 191 (2009).

45 C. Fricke, F. Hohls, C. Flindt, and R. J. Haug, High cumulants in the counting statistics measured for a quantum dot, Physica E 42, 848 (2010).

46 C. Fricke, F. Hohls, N. Sethubalasubramanian, L. Fricke, and R. J. Haug, High-order cumulants in the counting statistics of asymmetric quantum dots, Appl. Phys. Lett. 96, 202103 (2010).

47 Y. Komijani, T. Choi, F. Nichele, K. Ensslin, T. Ihn, D. Reuter, and A. D. Wieck, Counting statistics of hole transfer in a $p$-type GaAs quantum dot with dense excitation spectrum, Phys. Rev. B 88, 035417 (2013).

48 D. Kambly, C. Flindt, and M. Büttiker, Factorial cumulants reveal interactions in counting statistics, Phys. Rev. B 83, 075432 (2011).

49 D. Kambly and C. Flindt, Time-dependent factorial cumulants in interacting nano-scale systems, J. Comput. Electron. 12, 331 (2013).

50 By shifting the index $N$ in the sum, we can rewrite $M_{s}^{m}(t)=\sum_{N=0}^{\infty} s^{N}(N+m)^{(m)} P_{N+m}(t) / \sum_{N=0}^{\infty} s^{N} P_{N}(t)$ to see that negative powers of $s$ never occur. Therefore, also for $s=0$, the moments and thus the cumulants are well defined, $M_{0}^{m}(t)=m ! P_{m}(t) / P_{0}(t)$.

51 We first limit $N$ by some $N_{\max }$. Then $\mathcal{M}_{s}(z, t)=c \prod_{j}(z-$ $\left.z_{j}\right)^{\alpha_{j}}$ is a polynomial of order $N_{\max }$ and Eq. (2) with a finite sum is readily derived from performing the derivatives. At the end, we let $N_{\max } \rightarrow \infty$.

52 A. G. Abanov and D. A. Ivanov, Allowed Charge Transfers between Coherent Conductors Driven by a TimeDependent Scatterer, Phys. Rev. Lett. 100, 086602 (2008).

53 A. G. Abanov and D. A. Ivanov, Factorization of quantum charge transport for noninteracting fermions, Phys. Rev. B 79, 205315 (2009).

54 To lowest order in $t$, Eq. (3) takes the form $(-1)^{m-1} C_{s}^{m}(t)=(m-1) ![\langle N\rangle(t)]^{m}+\mathcal{O}\left(t^{m+1}\right) \geq 0$ for our system with $\langle N\rangle(t)=\frac{\gamma_{0 \uparrow} \gamma_{0 \downarrow}\left(\gamma_{\uparrow 0}+\gamma_{\downarrow 0}\right)}{\gamma_{0 \uparrow} \gamma_{\downarrow 0}+\gamma_{0 \downarrow}\left(\gamma_{0 \uparrow}+\gamma_{\uparrow 0}\right)} t$. Generalized factorial cumulants expressed by Eq. (2) are dominated by one zero at $-1 /\langle N\rangle(t)$. 